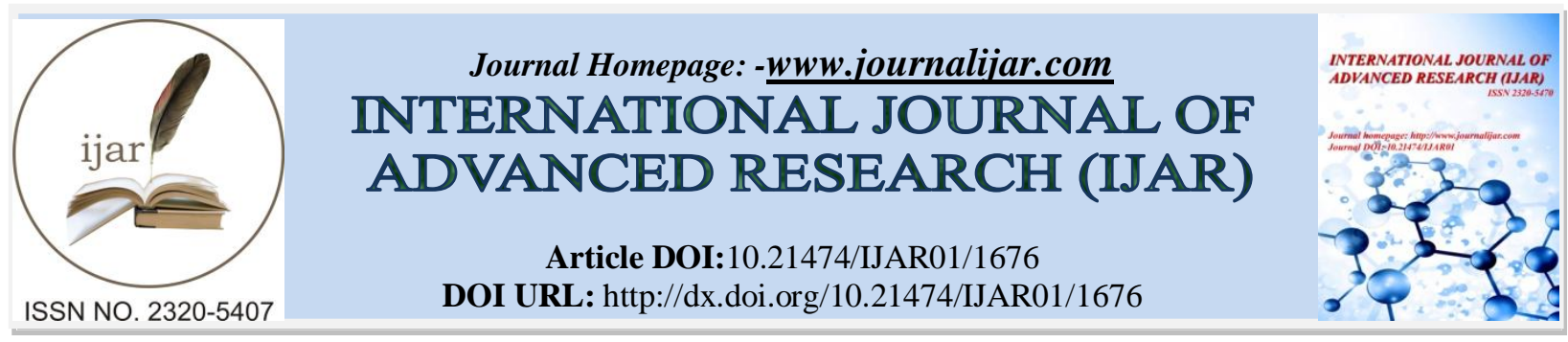

RESEARCH ARTICLE

\title{
NEEDLE STICK INJURY REPORTING BEHAVIOR AND RISK PERCEPTION AMONG SANITATION STAFF IN TERTIARY CARE HOSPITALS IN DELHI.
}

Puneet Chahar ${ }^{1}$, Vikrant R. Mohanty ${ }^{2}$, Aswini Y.B ${ }^{3}$, Vipul Yadav ${ }^{4}$ and Shekhar Grover ${ }^{5}$.

1. Post Graduate, Department of Public Health Dentistry, Maulana Azad Institute of Dental Sciences, New Delhi, India.

2. Associate Professor and HOD, Department of Public Health Dentistry, Maulana Azad Institute of Dental Sciences, New Delhi, India.

3. Assistant Professor, Department of Public Health Dentistry, Maulana Azad Institute of Dental Sciences, New Delhi, India.

4. Senior Resident, Department of Public Health Dentistry, Maulana Azad Institute of Dental Sciences, New Delhi, India.

5. Senior Resident, Department of Public Health Dentistry, Maulana Azad Institute of Dental Sciences, New Delhi, India.

\section{Manuscript Info}

(..........................

Manuscript History

Received: 12 July 2016

Final Accepted: 22 August 2016

Published: September 2016

Key words:-

\section{Abstract}

Hospital Sanitation Workers are exposed to a wide range of biological, chemical, and physical hazards. Needle stick injuries (NSI) constitute a recognized occupational hazard in hospitals. Therefore, the aim of the present study was to assess NSI Reporting Behaviour and Risk Perception among Sanitation Staff in Tertiary Care Hospitals.

\section{Introduction:-}

World Health Organization Health Report (2002) highlighted that there are 35 million health care workers out of which 2 million experience percutaneous exposure to infectious diseases each year. It was further noted that $37.6 \%$ of the Hepatitis B, $39 \%$ of Hepatitis C and 4.4\% of HIV/AIDS was observed among Health Care Worker (HCW) around the world which were inflicted due to needle stick injuries [1].

$\mathrm{HCW}$ are at increased risk of infection with Blood Borne Pathogen (BBP) because of occupational exposure to blood and other body fluids. Most exposure among HCW are caused by percutaneous injury with sharp objects contaminated with blood or body fluid [2]. Average risk of transmission after percutaneous exposure is approximately $0.3 \%, 6-30 \%$ and $1.8 \%$ for HIV, Hepatitis B and Hepatitis C. Needle Stick Injuries (NSI) can occur during usage, disposal of sharps or accidentally. It is important to dispose the sharps appropriately, otherwise can injure $\mathrm{HCW}$ who encounter them unexpectedly. Needle stick injuries present the single greatest risk to health care personnel and increasing awareness along with regular training in Universal Safety Precautions (USP) is required for prevention of nosocomial infection [3]. In spite of plethora of published literature and training programs to prevent these injuries, significant success has not been achieved in preventing NSI [4].

Public hospitals in India are in the process of revamping their images, earlier known for being unclean, and unhygienic these hospitals need to promote hygiene and sanitation. It faces a huge challenge in raising the quality of 
service and ensuring adequate hygiene and clean environment to patients and attenders while simultaneously gearing up its capabilities to tackle the changing disease patterns. This challenge needs to be addressed through a concerted effort of both public and private sectors along with supportive dialogue and understanding need of the system.

Sanitation workers performs a very critical function in health care. The main functions of this department is to ensure the overall cleanliness, ensuring maintenance of the facilities, biomedical waste management, infection control measures along with safety and security of the patients. These services if affected have an unswerving impact on health, comfort and morale of patients, staff and visitors. Public Hospitals need to take adequate and significant measures towards achieving this goal. Hence one of the methods to raise the quality and efficiency of services at the level of Sanitation is through Public Private Partnership (PPP). Currently the most acceptable and practical method is through outsourcing [5].

Outsourcing of sanitation services is expected to improve efficiency resulting in better patient and staff satisfaction, timely services, better management and control of services and materials in a time bound fashion at affordable cost that public hospitals can bear. But this truth is far from reality, as many agencies have mushroomed in the country without control and adequate permissions. These agencies have become hiring houses of lowly skilled, untrained and unverified employees which might be based on inadequate standard guidelines. Hence due to these reasons quality of services suffers in hospitals with varying health outcomes and lack of regular training of the staff results in inadvertent exposure to various occupational hazards [6]. The present study was conducted to assess Needle Stick Injury reporting behavior and risk perception among the sanitation staff in Tertiary Care Public Hospitals in Delhi.

\section{Materials and Methods:-}

Health care Infrastructure and services are being provided in the National Capital Territory of Delhi, India by a number of agencies like Central, State and Corporations through its network of hospitals and other specialized institutions. In addition to these, private sector is also contributing in provision of Health Care Services in an enormous way [5]. The present cross sectional study was carried out in two tertiary care public hospitals where sanitation staff was outsourced. The Tertiary care public hospitals were selected which delivered different health care services. The sample size of 150 was calculated by using the pilot study [prevalence of NSI (41.1\%)], considering precision of $20 \%$ and an additional false response rate of $10 \%$. Convenience sampling technique was used for selection of samples.

Ethical clearance was obtained from the parent Institution. Prior permission from the Head of the Institutions was obtained and informed consent was obtained from the participants. A structured self-designed questionnaire in local language (Hindi) was used to collect information about sociodemographic data, vaccination status, Risk perception towards NSIs, occurrence of NSIs in the past 6 months and reporting behavior. Validity and reliability was assessed using the pilot study and was found to be acceptable (Cronbach's alpha, $r=0.73$ ). Digital spreadsheets were made and data was analyzed using SPSS version11.5. Descriptive results were obtained and compared.

\section{Results:-}

With a response rate of $86.25 \%, 138$ respondents out of 150 completed the questionnaire. A majority of study population (73\%) were between the 20-30 years age group, most of them were males. About $74 \%$ of the sample population had secondary level education with only $9 \%$ either with primary education. The income of respondents ranged from Rs. 5,000-10,000. Work experience ranged from less than 1 year to 10 years, with a majority (77\%) of the subjects having 2-5years experience.

Around $93 \%$ of the respondents were aware of the injury from sharps and its prevention and agreed that they have been provided with some prior information about NSI. But when asked about the transmission of various blood borne diseases through NSI, most (51\%) of them considered AIDS was the only disease to be transmitted whereas $34 \%$ considered both AIDS and Hepatitis being transmitted. 34.7\% of the workers reported being vaccinated against Hepatitis B and only $21.1 \%$ were found to have undergone medical screening in the past 12 months. 
The incidence of the percutaneous NSI in the last six months among the respondents was about $30 \%$. The event reportedly occurred mostly during collecting the needle from the floor $(66 \%)$ whereas the other causes are described in Figure 01 (Fig. 01).

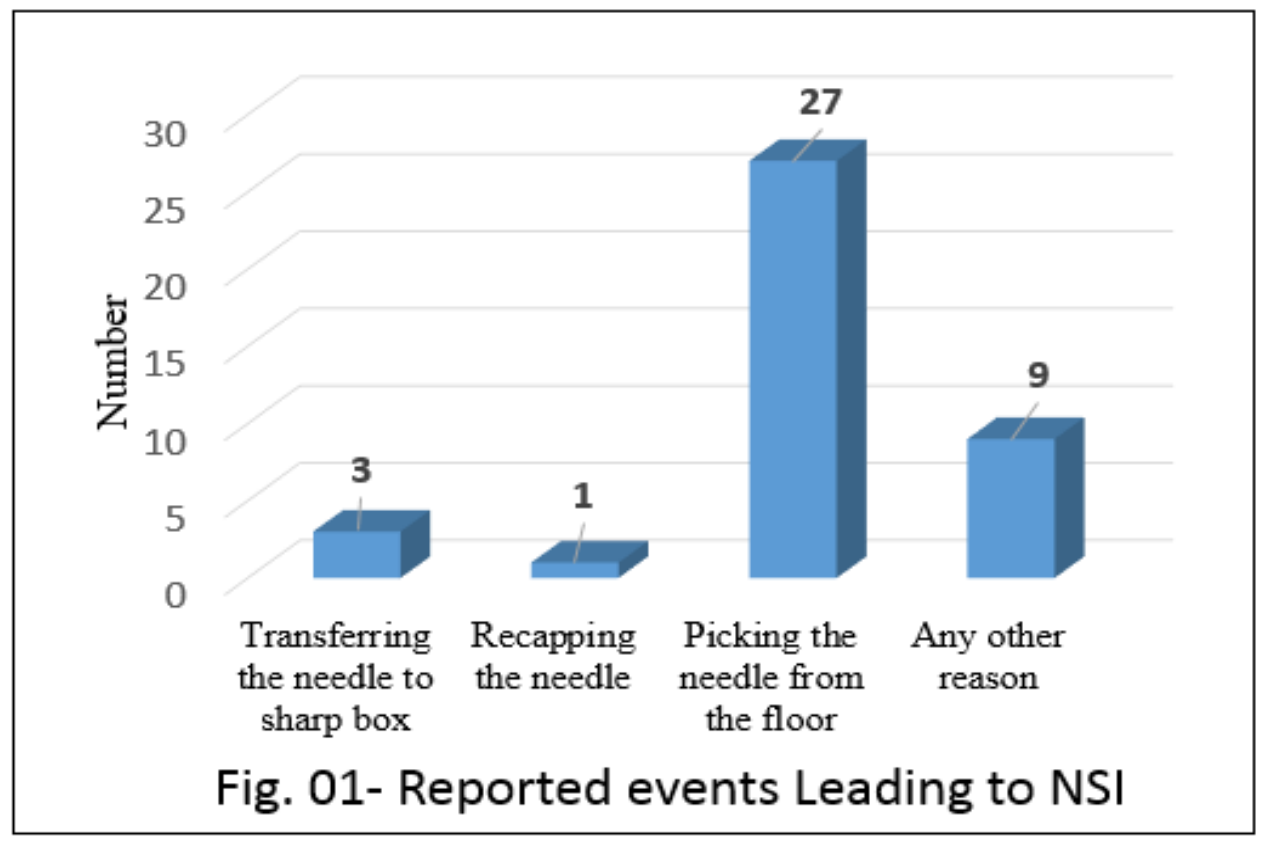

Carelessness (63\%) and pressure of completing work on time (32\%) were major reasons considered for NSIs. Washing the site with water after the prick was the most common measure (75\%) followed by washing with antiseptic and pressing the site as shown in the Figure 02 (Fig. 02).

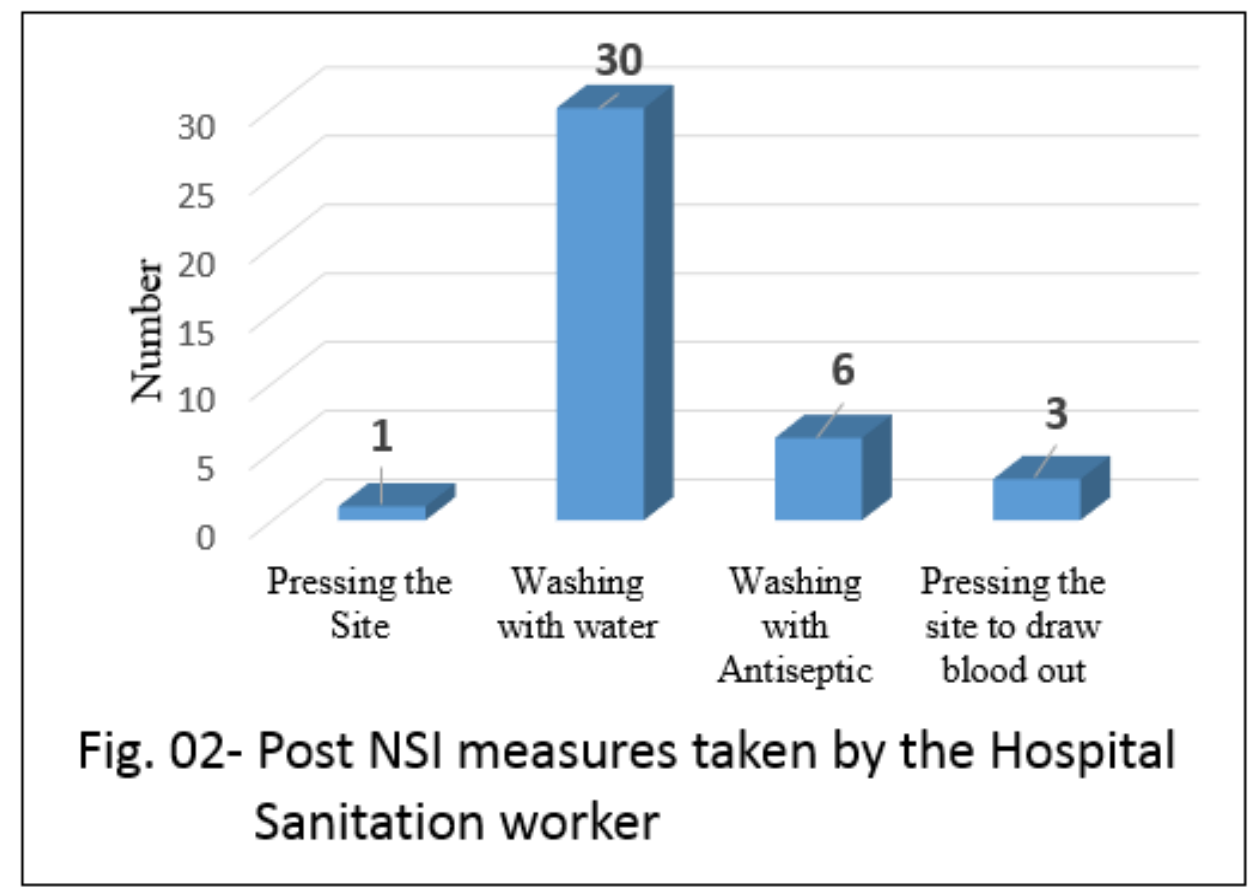

Around 95\% of the NSI were reported to the respective supervisors and Hospital officials as per protocol and 90\% received appropriate medical care in the form of Post Exposure Prophylaxis. 
The common reasons for under reporting were found to be fear of being diagnosed by HIV/AIDS (59.4\%), with other reasons as mentioned in Figure 03 (Fig. 03).

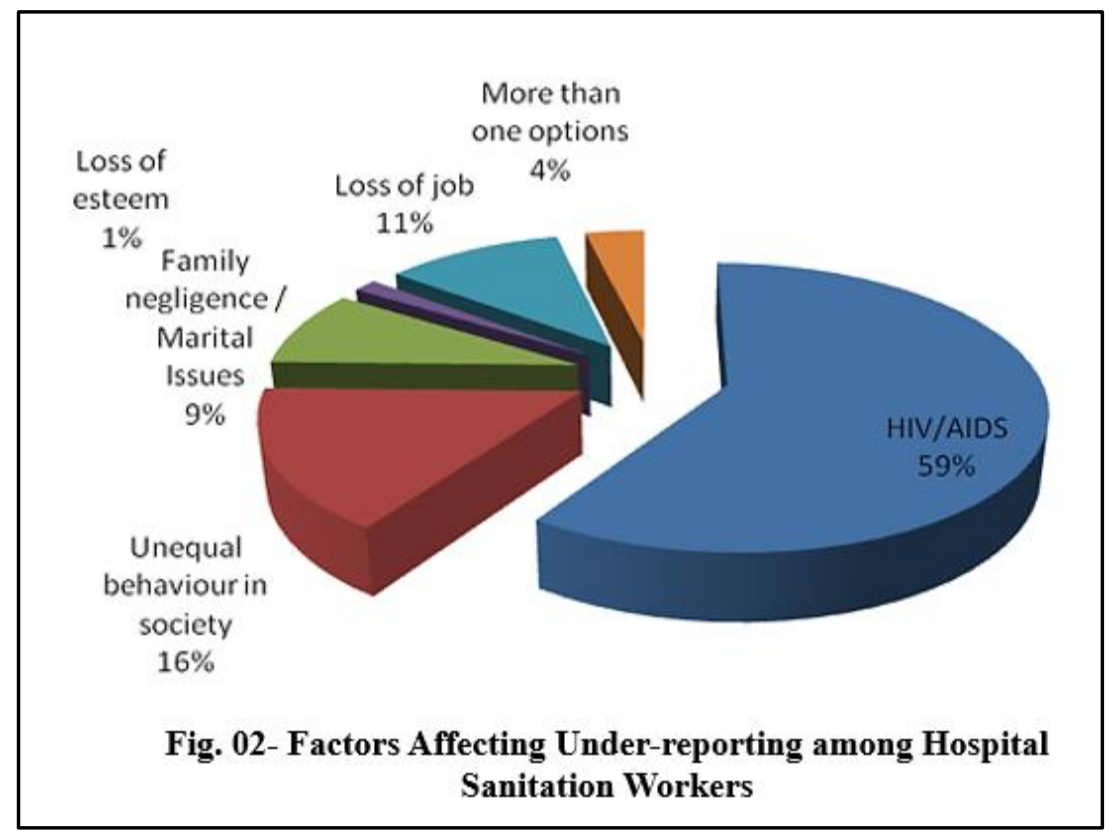

Majority of the respondents $(95.6 \%)$ expressed the need to be regularly updating and training to be carried out about NSI prevention and universal precautions. Around 65\% considered lectures and demonstrations as the method of education for orientation on risk reduction measures and post exposure management as an ideal method.

\section{Discussion:-}

Needle stick injury is a global phenomenon and regarded as one of the serious occupational hazards among health care workers. The Centers for Disease Control and Prevention (CDC) estimates that about 385,000 sharps-related injuries occur annually among health care workers in hospitals. Multiple studies have been done on doctors, medical students, nurses and other technical staff but none have focused on an equally vulnerable risk group of sanitation staff [7-10].According to a study which reviewed 316 reported NSIs, sanitation and laboratory personnel experienced highest incidence of NSIs [11].

In the present study, 93\% of the respondents were aware of the preventive measures to be taken while handling sharps. According to a study done in medical students, it was found that $99.3 \%$ of the students had knowledge about Blood Borne disease [10]. Only 34\% considered both HIV and Hepatitis being transferred by NSIs which was in accordance with previous studies [4, 12]. But in a study done by MaqboolAlam in Saudi Arabia among nurses and paramedical staff, only $20 \%$ were not aware of both HIV and Hepatitis being transferred by NSIs [13] whereas study conducted by Muralidhar et al. [14] among health care professionals found that almost all were aware of HIV and Hepatitis B and C being transferred by NSIs. Gaps in knowledge and awareness is evident in sanitation staff as compared to other health care professional. Outsourcing agencies often are hiring personals that have low level of education and no adequate training about potential occupational hazards.

The work environment of hospitals lead to an increased risk of acquisition of Blood borne pathogens such as HIV, hepatitis B and C. WHO estimates the global burden of disease from occupational exposure to be $40 \%$ of the hepatitis B and C infections and 2.5\% of the HIV infections among HCWs as attributable to exposures at work [1]. The immunization status for sanitation staff was found to be $22.5 \%, 16.3 \%$ and $92.3 \%$ in previous studies [4, $15,16]$ as compared to our study where $34.7 \%$ of the sample were vaccinated for Hepatitis B. Lack of awareness and lack of stringent implementation of vaccination protocol by the outsourcing companies may be some of the reasons for low immunization status.

Moreover the low reporting should not be considered same as low incidence of NSIs, which may be due to a global phenomenon of Underreporting of sharp injury. Lakbala et al. reported incidence of only $22.8 \%$ in 1 year for sanitation workers [16]. When compared with the incidence among other health professionals like nurses, doctors, 
laboratory technicians and paramedical staff, it was observed consistently lower among sanitation staff [17-19, 13]. A low Incidence rate in the present study may be because of underreporting of the NSI events, lack of training, tight work schedule and low awareness. Underreporting may also be attributed to lower education status, different cultural backgrounds, ignorant attitude of administrators and probably language barriers [17, 20, 21]. Though the extent of underreporting in the present study was unknown but further studies could explore rates of underreporting sharp injuries and raise possible suggestions as to counter the current situation.

The fear of being diagnosed by HIV/AIDS (59.4\%), followed by stigma and discrimination in the society (15.9\%) could be causes of underreporting among the present participants. The results expose the fact that transmission of HIV, associated stigma and discrimination are still high in the society. Stigma and its outcome i.e. Discrimination are common human reaction to people contracting diseases like leprosy, tuberculosis, mental illness and STDs. These barriers remain an obstacle in the efforts to mobilize communities and professional groups.

Most of the events (66\%) occurred while picking up the sharp from the floor which was in accordance with the study done by Bindra et al. [4] where manipulation of sharps was found to be leading cause of NSIs. In other studies carried out among health care workers it was observed that most of the incidents occurred while recapping the needle $[14,22,10]$. In the current negligent and improper disposal of the needle by the sanitation workers may be the reason. When asked about the predisposing factor that led to NSI, majority (63\%) of the considered carelessness to be the major cause of sharp injuries. Previous studies have shown fatigue with long working shifts were identified as reasons for increased risk of NSI [9, 23]. Understaffing, increasing workload, and working long hours may be responsible for fatigue, stress, emotional exhaustion, carelessness, which may ultimately result in poor compliance with precautions at work.

\section{Conclusion:-}

Needle stick injuries are more than just a quality indicator in health care today. Awareness and risk of transmission of diseases through NSI are universal for all health care professionals.

Outsourcing is a superior measure towards improved efficiency, better management and control of services. But Public Hospitals should develop stringent and effective policies for outsourcing vital services. Hospital administrators should further reinforce these policies by constant supervision and regular monitoring through clinical audits. NSI can be prevented by providing continued education, standard operating procedures and effective training. Induction programs for new entrants and creation of role models as team building exercises should be a regular element of the training.

Though the study had its novelty in assessing the needle stick injury behaviour in exclusively sanitation workers but there are limitations to this study which should also be taken into account. Firstly, convenience sampling method has been used and the study relies mainly on the recall/memory of the respondents, which could lead to recall bias.

\section{Recommendations:-}

* Intensive, Periodic training for safer handling and disposal of sharps for all the staff.

* Universal precautions and should be adopted to prevent and reduce hazards from NSI to minimum.

* Incorporating a culture of mandatory reporting of NSIs and regular supervision.

* Schedule of regular medical examination and vaccinations programs be drawn.

* Standard recruitment protocol for outsourcing staff and regular evaluation of work. 


\section{References:-}

1. WHO Health Report. [Internet]. 2015 [Cited 2015 Sept 20]; Available from: URL:http://www.who.int/occupational_health/topics/needinjuries/en/.

2. Prüss-Üstün A, Rapiti E, Hutin Y. Estimation of the global burden of disease attributable to contaminated sharps injuries among health-care workers. Am. J. Ind. Med. 2005;48: 482-90.

3. Rele M, Mathur M, Turbadkar D. Risk of needle stick injuries in health care workers-A report. Indian J med Microbiology 2002;20:206-7

4. Bindra S, Reddy KVR, Chakrabarthy A, Chaudhary K. Awareness about needle stick injures and sharps disposal: a study conducted at army college of dental sciences. J Maxillofac Oral Surg. 2013;13(4):419-24.

5. Economic Survey of Delhi 2012-2013. [Internet]. 2014 march 23 [Cited 2015 Sept 20]; Available from: URL:http://delhi.gov.in/wps/wcm/connect/doit_planning/Planning/Misc./Economic+Survey+of+Delhi+201213.

6. Roberts JG, Henderson JG, Olive LA, Obaka D. A Review of Outsourcing of Services in Health Care Organizations. Journal of Outsourcing and Organizational Information Management. 2013 Jan 1;2013:1.

7. Khurl-Bulos NA, Toukan A, Mahafzah A, Al Adham M, Faori I, Khader IA, Rumeileh ZIA. Epidemiology of needlestick and sharp injuries at a university hospital in a developing country: a 3-year prospective study at the Jordan University Hospital, 1993 through 1995. American Journal of Infection Control 1997;25:322-9.

8. Askarian M, Malekmakan L. The prevalence of needle stick injuries in medical, dental, nursing and midwifery students at the university teaching hospitals of Shiraz, Iran. Indian J Med Sci 2006;60:227-32.

9. Nsubuga FM, Jaakkola MS. Needle stick injuries among nurses in sub-Saharan Africa. Tropical medicine \& international health 2005;10:773-81.

10. Norsayani MY, Noor Hassim I. Study on incidence of needle stick injury and factors associated with this problem among medical students. Journal of occupational health 2003;45:172-8.

11. McCormick RD, Maki DG. Epidemiology of needle-stick injuries in hospital personnel. The American Journal of Medicine 1981;70:928-32.

12. Pandit NB, Mehta HK, Kartha GP, Choudhary SK. Management of bio-medical waste: Awareness and practices in a district of Gujarat. Indian J Public Health 2005;49:245-7.

13. Alam M. Knowledge, attitude and practices among health care workers on needle-stick injuries. Ann Saudi Med 2002;22:396-9.

14. Muralidhar S, Singh PK, Jain RK, Malhotra M, Bala M. Needle stick injuries among health care workers in a tertiary care hospital of India. Indian J Med Res 2010;131:405-10.

15. Yacoub R, Al Ali R, Moukeh G, Lahdo A, Mouhammad Y, Nasser M. Hepatitis B vaccination status and needlestick injuries among healthcare workers in Syria. Journal of global infectious diseases 2010;2:28-34.

16. Lakbala.P, Azar. F.E. Kamali.H. Needle stick and sharps injuries among housekeeping workers in hospitals of Shiraz, Iran. BMC Research Notes 2012;5:276-80.

17. Al-Turki KA, Abu-Gad HA. Frequency of and prevention measures for needle-stick injuries among hospital healthcare workers in saudiarabia. Journal for Healthcare Quality 2000;22:23-28.

18. Chakravarthy M, Singh S, Arora A, Sengupta S, Munshi N. The epi-net data of four Indian hospitals on incidence of exposure of healthcare workers to blood and body fluid: a multicentric prospective analysis. Indian J Med Sci. 2010, 64(12):540-8.

19. Jayanth ST, Kirupakaran H, Brahmadathan KN, Gnanaraj L, Kang G. Needle stick injuries in a tertiary care hospital. Indian journal of medical microbiology 2009;27:44-7.

20. Ng LN, Lim HL, Chan YH, \& Bin Bachok D. Analysis of sharps injury occurrences at a hospital in Singapore. International journal of nursing practice 2002;8:274-81.

21. Efstathiou G, Papastavrou E, Raftopoulos V, \&Merkouris A. Prevalence of Occupational Exposure to Pathogens and Reporting Behaviour among Cypriot Nurses. Int J Caring Sci 2013;6:420-30.

22. Sharma R, Rasaniya SK, Verma A, Singh S. Study of prevalence and response to needles stick injuries among health care workers in a tertiary care hospital in Delhi. India. Indian J Community Med. 2010;35:74-7.

23. Sharma A, Gur R, Bhalla P. Study on prevalence of needle stick injury among health care workers in a tertiary care hospital in New Delhi: A two-year review. Indian J Public Health 2012;56:101-3. 